\title{
Experimental Observation of "Shadowing" in Optical Transition Radiation
}

\author{
R. Kieffer, ${ }^{1}$ M. Bergamaschi, ${ }^{1,2}$ E. Bravin, ${ }^{1}$ W. Farabolini, ${ }^{1}$ P. Karataev, ${ }^{2}$ T. Lefevre, ${ }^{1}$ and S. Mazzoni ${ }^{1, *}$ \\ ${ }^{1}$ CERN, CH-1211 Geneva 23, Switzerland \\ ${ }^{2}$ John Adams Institute at Royal Holloway, University of London, Egham, Surrey TW20 OEX, United Kingdom
}

(Received 7 July 2017; published 28 February 2018)

\begin{abstract}
We report the observation of shadowing between two optical transition radiation (OTR) sources from a $205 \mathrm{MeV}$ electron beam. The total optical intensity is measured as a function of the distance $d$ between the sources, covering the range $0<d<4 L_{v}$, where $L_{v}$ is the formation length of the particles. Data show that the total optical intensity starts decreasing due to shadowing when $d$ approaches $L_{v}$ until it becomes undetectable for very short distances $d / L_{v} \rightarrow 0$. A model based solely on interference between the two OTR sources is in good agreement with experimental data. To the knowledge of the authors this is the first systematic experimental observation of shadowing in OTR.
\end{abstract}

DOI: 10.1103/PhysRevLett.120.094802

Transition radiation (TR) refers to the emission of broadband electromagnetic radiation that takes place when a charged particle crosses the boundary between two media with different dielectric properties. Originally predicted by Ginsburg [1] in 1946, TR has been studied experimentally during the last 60 years. The optical frequency band of TR (we will refer to it as OTR) is routinely used as a beam diagnostic technique in particle accelerators $[2,3]$ where the spatial, temporal, or angular characteristics of OTR are measured. Extensive theoretical and experimental studies were launched since to fully understand OTR. Assuming that the transition radiation field is not a point source but expands transversely similarly to the particle field (i.e., $\gamma \lambda / 2 \pi$, where $\gamma$ is the Lorentz factor and $\lambda$ the radiation wavelength) Verzilov [4] introduced the concept of a prewave zone. It corresponds to distances for which the angular distribution of the electromagnetic field is affected by the transverse dimension of the source. Transition radiation in the prewave zone was then studied experimentally [5] showing good agreement with theoretical expectations. Far field conditions can only be considered valid for distances from the source much larger than $\gamma^{2} \lambda / 2 \pi$, which implies that beam diagnostics based on TR radiation using long wavelength or for very high beam energies will be perturbed by prewave zone effects [6,7]. A mitigation technique consisting of putting the detector in the back focal plane of a focusing lens [8] was then proposed to suppress prewave zone uncertainties and an experimental validation was performed successfully soon after at ATF-KEK [9].

Published by the American Physical Society under the terms of the Creative Commons Attribution 4.0 International license. Further distribution of this work must maintain attribution to the author(s) and the published article's title, journal citation, and DOI.
While TR emission from a single source is relatively well known and understood, there exists a class of beam instruments and detectors that requires the use of multiple TR sources. Key to a correct interpretation of multiple TR emission is the concept of formation length described by Garibyan in Ref. [10] as the distance over which the phase difference between the radiation field and the particle field changes by $1 \mathrm{rad}$ and is defined by

$$
L_{v}=\frac{\lambda}{2 \pi} \beta(1-\beta \cos \theta)^{-1},
$$

where $\theta$ is the angle with respect to the particle trajectory and $\beta$ the ratio of the particle velocity to the speed of light. For relativistic particles, the formation length of forward transition radiation (FTR, i.e., along the particle trajectory) is proportional to $\gamma^{2} \lambda$ and can extend to large distances, whereas for backward transition radiation (BTR, i.e., along the direction of specular reflection from the target surface), it is typically limited to distance of the order of the wavelength [11].

Even though the formation length is defined by a parameter similar to the prewave zone, one should not mix up these two concepts. The former is defines a length over which the particle field is restored, while the latter defines a distance at which TR is purely angular. When two or more TR sources are present, it is predicted that the total emitted power can be enhanced if the distance between TR sources is longer than $L_{v}$, respectively, or when $\delta>1$ when we define $\delta=d / L_{v}$ as the distance between radiators normalized by formation length. Some aspects of multi-TR emission were studied in the past years. The enhancement of $x$-ray TR in a stack of thin foils was investigated theoretically [12] and experimentally [13] in the 1970s. The angular distributions in the far-field region from electrons of $70 \mathrm{MeV}$ and distances between foils down 
to $2 \mathrm{~mm}(\delta=1.2-15)$ were studied in the pioneering work of Wartski [14] on optical transition radiation interference (OTRI). Several experiments on interferometry $[15,16]$ were conducted more recently in similar conditions, i.e., with $\delta$ values of 3-23 and 10, respectively. They all reported beam measurements well in agreement with Wartski's predictions and proposes the use of such an interferometer for beam size, emittance, and energy measurements. More recently, advanced beam diagnostic techniques using both optical transition and diffraction radiation [17-19] require the use of two thin metallic foils or slits separated by a distance much shorter than the formation length $(\delta \ll 1)$. In this case, pronounced suppression of the TR field, also referred to as the shadowing effect $[20,21]$, is predicted to occur. A first observation of shadowing was recently performed by Naumenko [22] in 2010 in the $\mathrm{mm}$ wave regime using coherent diffraction radiation. A reduction of the light emitted by a two-slits assembly by more than a factor of 5 for relative distance $\delta$ as short as 0.1 was reported. However, at such long wavelengths the measurements were performed in extreme prewave zone and diffraction conditions, which undoubtedly introduced additional uncertainty. To understand the effect of shadowing and its practical consequences, detailed investigations to the phenomena as a function of $\delta$ are required. To date, a systematic measurement of shadowing as a function of $\delta$ for OTR is still lacking. We report in this Letter the first observation of a strong shadowing effect in the visible domain using incoherent optical transition radiation interference (OTRI). The results are in good agreement with Wartski's prediction, and elucidate the link between the suppression of optical power and the appearance of interference lobes at progressively higher angles when $\delta \rightarrow 0$.

Experiments were conducted in the CALIFES beam line of the CLIC Test Facility 3 (CTF3) at CERN, where electrons are accelerated up to an energy of $205 \mathrm{MeV}$, with a corresponding formation length in the optical region of $L_{v}(\lambda=500 \mathrm{~nm}, \theta=0)=25 \mathrm{~mm}$ [see Eq. (1)]. A schematic drawing of the setup is shown in Fig. 1. A $100 \mu \mathrm{m}$ thick aluminium foil (a) and a $200 \mu \mathrm{m}$ thick aluminumcoated silicon screen (b) are mounted on a linear actuator that moves the screen assembly across the beam path. The assembly is designed to have an angle of $90^{\circ}$ between the screens so that the distance traveled by the particle beam between the two screens $d$ can be changed depending on the position of the screen assembly within a range $0<d<36 \mathrm{~mm}$. Screen (b) is longer than (a) by $9 \mathrm{~mm}$, allowing the particle beam to solely cross screen (b), therefore producing backward single-screen OTR as a reference. Because of the $45^{\circ}$ orientation of both screens with respect to the electron beam, the TR signal is emitted orthogonally from the beam path through a borosilicate glass viewport (not shown in Fig. 1). The whole optical setup is mounted on a motorized translation stage allowing the control of its transverse position with respect to the target assembly. Indeed, when the screen assembly is moved across the beam, the area where screen (b) intercepts the beam (that is the OTR source) moves along the beam path. Given the narrow (of the order of $1 / \gamma=2.5 \mathrm{mrad}$ ) angular distribution of OTR light, to avoid any vignetting effect the typical conical light emission of OTR must coincide with the optical axis of the instrument. Therefore, a transverse scan of the optical imaging system until the light intensity reaching the sensor is maximized is needed whenever the distance between screens is changed. The measurements presented in this document took place during two shifts campaign dedicated to OTR imaging and angular distributions. Figure 1 shows the setup for angular measurements. A 12 bit, 1/3 inch CCD sensor $(f)$ is placed in the backfocal plane of a $f=40 \mathrm{~mm}, 1^{\prime \prime}$ diameter air-spaced doublet (e) to reproduce far-field, angular distribution, after passing through a set of band pass filters (400 or $650 \mathrm{~nm}$; bandwidth $40 \mathrm{~nm}$ ) (d). The focus is adjusted prior to the experiment, by sending a laser centered inside the beam pipe following the electron beam path. The system is designed so to record an angular range $-42<\theta<42 \mathrm{mrad}$, with a resolution of $\cong 94 \mu \mathrm{rad} / \mathrm{pixel}$. A pellicle beam splitter (c) sends approximately $3 \%$ of the light to a camera for monitoring purposes (h). Not shown in Figure 1 is the imaging configuration, where the cemented doublet is replaced with a $35 \mathrm{~mm}-\mathrm{F} 1.6$ camera lens to form an image of screen (b) onto the CCD sensor plane. As the angular pattern is spread over a much larger CCD sensor area than for imaging, the number of electron bunches in the train for angular measurements was increased by an order of magnitude with respect to imaging ones to partially recover light intensity and, in turn, to improve the signal to noise ratio. An overview of spatial

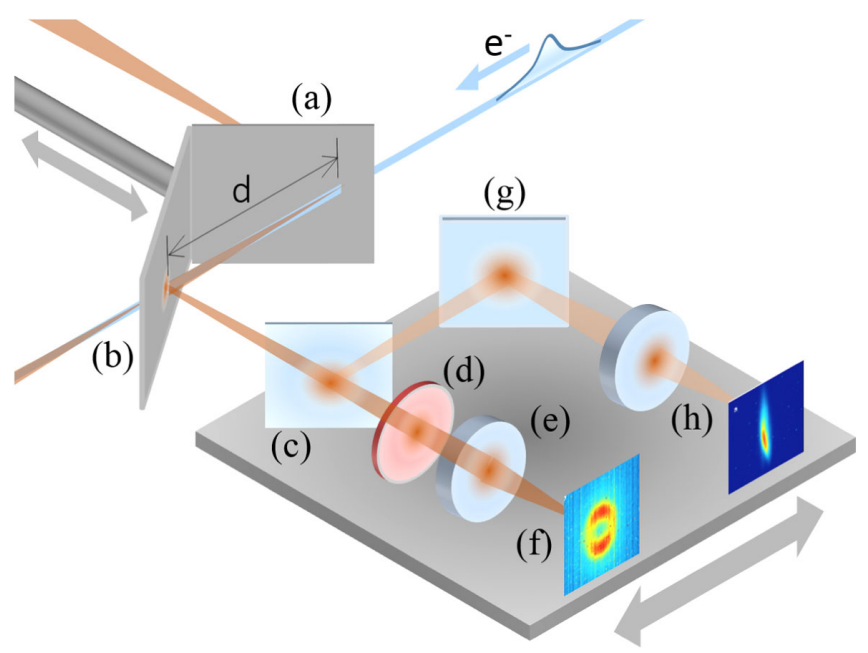

FIG. 1. Sketch of the OTRI setup on the CALIFES beam line (not to scale). Screens (a) and (b) are mounted on a linear translator that determines the interscreen distance $d$. The optical instrument can translate along the beam direction to center the OTR emission cone on the optical axis. 
and angular distribution images is shown in Fig. 2. The top row shows the intensity spatial distribution at the surface of screen (b) of Fig. 1, bottom row the intensity angular distribution at corresponding $\delta$ values. Images (a), (b) are acquired at a distance of $d=17.8$, (c), (d) at $d=7.7$, (e), (f) at $d=2.5$, and (g), (h) at $d=0.75 \mathrm{~mm}$, corresponding to $\delta$ values of $1.11,0.47,0.16$, and 0.05 respectively. The horizontal profile of the images is shown on subplot (i). Subplot (j) shows the four transverse intensity profiles. One may see that in the imaging case the image brightness is decreasing while the width of the distribution almost remains unchanged. On the other hand the angular distribution (bottom row) gets broader for decreasing values of $\delta$. Such a broadening with respect to a single screen distribution was observed by Ref. [14]. It illustrates that pure interference between two sources responsible for the intensity redistribution appears only when the radiation amplitudes at the detector plane from two sources are comparable. This is not the case for imaging scenario until the distance between them becomes very short. We have recorded the total OTRI intensity from the integral of the spatial distribution for two wavelengths, 400 and $650 \mathrm{~nm}$, see Fig. 3. On the bottom plot, the OTRI curves are normalized to the intensity of the single screen and plotted as a function of the normalized distance $\delta$. Starting from the right-hand side ( $\delta>2.6$ for $400 \mathrm{~nm}, \delta>4.2$ for $650 \mathrm{~nm}$ ), where the signal is generated solely by the second screen (b), the total intensity reaches twice the reference value as soon as the beam crosses both screens (a) and (b), as predicted in Ref. [14]. The intensity curves remain approximately constant until $\delta \approx 1$, where shadowing starts to appear and the total emitted optical intensity drops abruptly. At distance $\delta<1$ the FTR severely interferes with the electron field partially canceling it out. It results in a reduced effect of polarization at the second target and decrease of the produced light intensity. However, if we compare OTRI intensity at different wavelengths, the curves overlap only when plotted as a function of normalized distance (see the difference between top and bottom plots). This illustrates that the photon yield is not only reduced but shifted towards higher frequencies.

We have compared experimental data with analytical formulas for the far-field distribution per unit frequency $\omega$ over a solid angle $d \Omega=\sin \theta d \theta d \phi[14,23]$ :

$$
\frac{d I}{d \omega}=F(\omega) \frac{e^{2} v^{2} \sin ^{3} \theta d \theta d \phi}{\pi^{2} c^{3}\left[1-\left(v^{2} / c^{2}\right) \cos ^{2} \theta\right]^{2}}\left|1-e^{-i \delta}\right|^{2},
$$

where $F(\omega)$ is the surface reflectivity, $e$ the elementary charge, $c$ the speed of light in vacuum, $v$ the particle speed, $\theta$ and $\phi$ are the polar and azimuthal angles, respectively. Equation (2) attributes the decrease of total emitted power to the progressive destructive interference between the two OTR sources. What is shown on Fig. 3 (black solid line) is the integral of Eq. (2) over the same experimentally accessible angular range, normalized to the single screen total emission calculated with the same formula. Analytical predictions are in very good agreement with experimental data. This seems to suggest that a model solely based on optical interference between two identical OTR sources
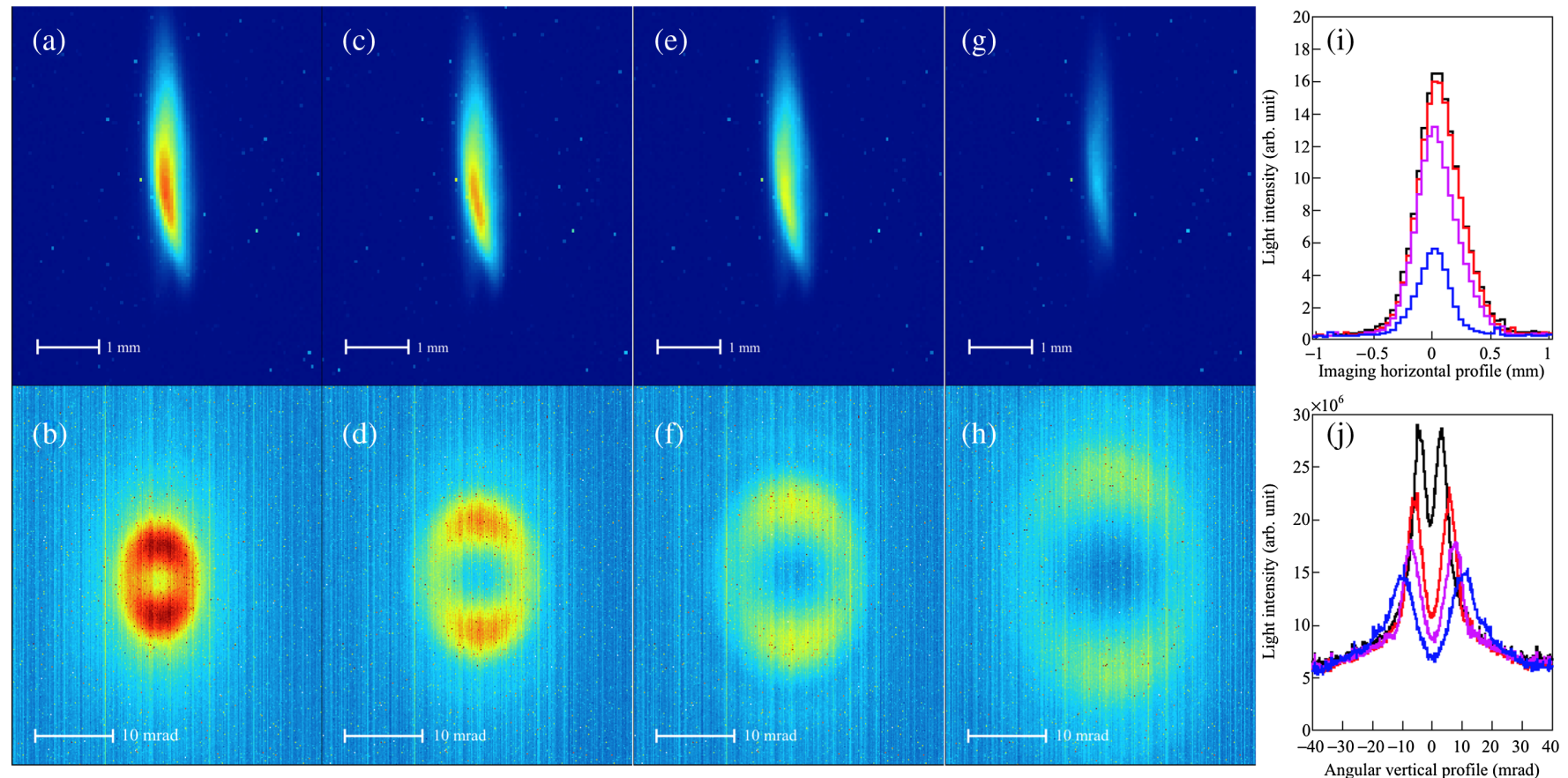

FIG. 2. Example of spatial (top row) and angular (bottom row) intensity distribution at $650 \mathrm{~nm}$ at $\delta$ values of $1.11,0.47,0.16$, and 0.05 starting from the leftmost figures. Subplots (i) and (j) show the correspondent transverse profiles. 

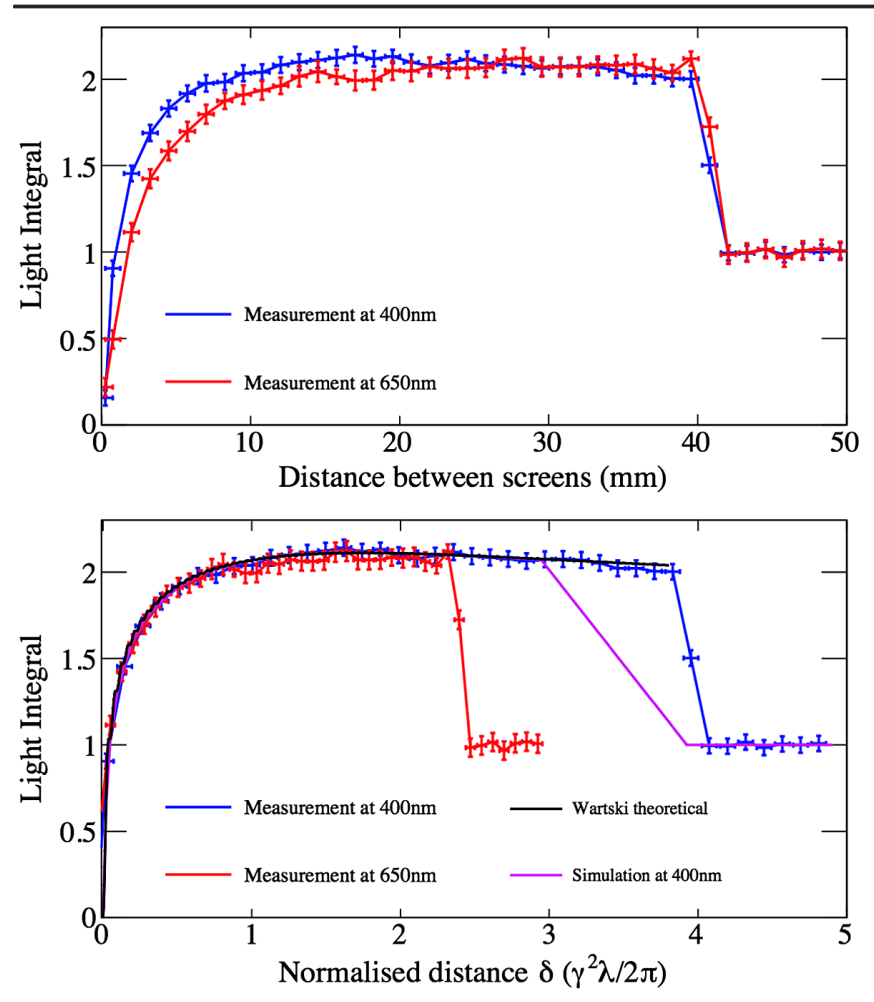

FIG. 3. OTRI intensity, normalized to the single screen OTR intensity as a function of the interscreen distance, for the wavelengths 400 (blue line) and 650 (red line) nm (top). Bottom plot is the same quantity plotted as a function of the normalized distance $\delta$. Experimental results are in very good agreement with analytical results from Ref. [14] (black line) and with numerical results.

separated by a distance $d$ and with a phase relation $\delta$ between them can correctly describe shadowing. This is remarkable as the physical process per se is not the optical interference between the two OTR sources but between the forward OTR emitted by the first screen and the electromagnetic field associated with the electrons. The latter is not a purely transverse electromagnetic field and therefore, only to a degree of approximation can be considered a radiation field (i.e., light). By adopting this approach, shadowing is the interference between the forward OTR photons and the quasiphotons associated with the moving electron (see Refs. [21,24]). Any radiator downstream of the first one at a normalized distance of $\delta<1$ will be in its shadow. At a distance $\delta \ll 1$, the FTR field almost completely cancels out the field of the electron, which is a region where no radiation is produced. At a distance $\delta \gg 1$, the electron field will be completely restored to its original value and is completely separated with the FTR field.

The validity of a purely optical model for shadowing is further confirmed by optical simulations, performed with Zemax OpticStudio $[25,26]$, of the propagation of the forward OTR field from the first screen to the second one using near-field diffraction formulas. The resulting field is overlapped with the backward OTR field generated by the beam at the input face of the second screen. The phase difference $e^{i \delta}$ is added to account for the time of flight difference between the particles and the forward OTR field when propagating between the two screens. The integral of the resulting angular curves over the angular range accessible to the instrument are plotted on Fig. 3 (solid purple line) for $400 \mathrm{~nm}$, showing very good agreement with both the experimental and analytical data.

The far-field, angular intensity distribution of OTRI has also been recorded at wavelengths of 650 and $400 \mathrm{~nm}$ over the entire range of normalized distances $0<\delta<2.4$ and $0<\delta<4$, respectively. While angular OTRI data do not contain information about the beam transverse spatial distribution, they are affected by its angular dispersion. Under the assumption that the latter takes the shape of a Gaussian distribution, the angular distribution of OTRI light can be written as

$$
I(\Omega, \omega) \propto \frac{d^{2} I}{d \Omega d \omega} \otimes e^{\frac{\vartheta_{h}^{2}+\vartheta_{v}^{2}}{2\left(\sigma_{h}^{2}+\sigma_{v}^{\prime 2}\right)}}
$$

where $\sigma_{h}^{\prime}$ and $\sigma_{v}^{\prime}$ are the horizontal and vertical divergence, respectively, $\otimes$ represents the convolution, and $\left(d^{2} I / d \Omega d \omega\right)$ is the zero-beam size distribution as written in Eq. (2). In our case, even though the horizontal and vertical divergence of the electron beam are not measured, it can be deduced from the bottom row of Fig. 2 that the horizontal one is more pronounced than the vertical one. In general, interference fringes for moderate and high values of $\delta$ are so fine that the optical instrument is not able to resolve them due to its finite resolution. In addition, electron beam divergence and color filter bandwidth contribute to their smearing. In the region $\delta \gg 1$, the pattern appears as the sum of two OTR angular distributions, with main lobes at $1 / \gamma \approx 2.5 \mathrm{mrad}$ and pronounced side tails. At decreasing values of $\delta$, the two sources become progressively out of phase, reaching complete opposition for $\delta=0$. This translates into a progressively strong destructive interference that suppresses the OTR lobes and shifts the signal over larger angles, with an ever decreasing intensity, as can be clearly seen in subplot (j) of Fig. 2. This mechanism elucidates the resulting loss of detectable signal, which is what is referred to as shadowing.

In conclusion, we have performed for the first time clear measurement of shadowing in OTR with a $205 \mathrm{MeV}$ electron beam. The resulting optical intensity has been measured as a function of the distance between the two OTR sources by forming an image of the second screen onto a CCD camera. The integrated intensity is twice the one emitted by a single OTR source for distances larger than the particles formation length, and decreases progressively for shorter distances until becoming undetectable.

The authors thank X. Artru for interesting discussions and suggestions. 
*Corresponding author. stefano.mazzoni@cern.ch

[1] V. Ginzburg and I. M. Frank, Zh. Eksp. Teor. Fiz. 16, 15 (1946).

[2] D. Rule, Nucl. Instrum. Methods Phys. Res., Sect. B 24-25, 901 (1987).

[3] M. Castellano, M. Ferrario, S. Kulinski, M. Minestrini, P. Patteri, F. Tazzioli, L. Catani, L. Gregorini, and S. Tazzari, Nucl. Instrum. Methods Phys. Res., Sect. A 357, 231 (1995).

[4] V. Verzilov, Phys. Lett. A 273, 135 (2000).

[5] M. Castellano, V. Verzilov, L. Catani, A. Cianchi, G. D'Auria, M. Ferianis, and C. Rossi, Phys. Rev. E 67, 015501 (2003).

[6] D. Karlovets and A. Potylitsyn, Nucl. Instrum. Methods Phys. Res., Sect. B 266, 3738 (2008).

[7] G. Naumenko, Nucl. Instrum. Methods Phys. Res., Sect. B 227, 87 (2005).

[8] P. Karataev, Phys. Lett. A 345, 428 (2005).

[9] P. Karataev, S. Araki, A. Aryshev, G. Naumenko, A. Potylitsyn, N. Terunuma, and J. Urakawa, Phys. Rev. ST Accel. Beams 11, 032804 (2008).

[10] G. Garibyan, J. Exp. Theor. Phys. 10, 372 (1960).

[11] X. Artru, R. Chehab, K. Honkavaara, and A. Variola, Nucl. Instrum. Methods Phys. Res., Sect. B 145, 160 (1998).

[12] X. Artru, G. B. Yodh, and G. Mennessier, Phys. Rev. D 12, 1289 (1975).

[13] L. C. L. Yuan, C. L. Wang, H. Uto, and S. Prünster, Phys. Rev. Lett. 25, 1513 (1970).

[14] L. Wartski, S. Roland, J. Lasalle, M. Bolore, and G. Filippi, J. Appl. Phys. 46, 3644 (1975).
[15] A. Lumpkin, R. Feldman, D. Feldman, S. Apgar, B. Carlsten, R. Fiorito, and D. Rule, Nucl. Instrum. Methods Phys. Res., Sect. A 285, 343 (1989).

[16] C. Couillaud, A. Loulergue, and G. Haouat, in Proceedings of the Fifth European Particle Accelerator Conference, Sitges (Barcelona) (1996), p. 1594.

[17] P. Karataev, S. Araki, R. Hamatsu, H. Hayano, T. Muto, G. Naumenko, A. Potylitsyn, N. Terunuma, and J. Urakawa, Phys. Rev. Lett. 93, 244802 (2004).

[18] A. Cianchi, V. Balandin, M. Castellano, E. Chiadroni, L. Catani, N. Golubeva, K. Honkavaara, G. Kube, and M. Migliorati, New J. Phys. 16, 113029 (2014).

[19] R. B. Fiorito and D. W. Rule, Nucl. Instrum. Methods Phys. Res., Sect. B 173, 67 (2001).

[20] X. Artru and C. Ray, Nucl. Instrum. Methods Phys. Res., Sect. B 266, 3725 (2008).

[21] G. Naumenko, Y. Popov, and M. Shevelev, J. Phys. Conf. Ser. 357, 012005 (2012).

[22] G. Naumenko, X. Artru, A. Potylitsyn, Y. Popov, L. Sukhikh, and M. Shevelev, J. Phys. Conf. Ser. 236, 012004 (2010).

[23] B. M. Bolotovskii and E. A. Galst'yan, Phys. Usp. 43, 755 (2000).

[24] S. Dobrovolsky and N. Shul'ga, Nucl. Instrum. Methods Phys. Res., Sect. B 201, 123 (2003).

[25] T. Aumeyr, M. G. Billing, L. M. Bobb, B. Bolzon, E. Bravin, P. Karataev, K. Kruchinin, T. Lefevre, and S. Mazzoni, Phys. Rev. ST Accel. Beams 18, 042801 (2015).

[26] B. Bolzon, A. Aryshev, T. Aumeyr, S. Boogert, P. Karataev, K. O. Kruchinin, T. Lefevre, S. Mazzoni, L. Nevay, M. Shevelev et al., Phys. Rev. ST Accel. Beams 18, 082803 (2015). 